\title{
PRIMA Astrometry Operations and Software
}

Eric J. Bakker ${ }^{a}$, Andreas Quirrenbach ${ }^{a}$, Robert N. Tubbs ${ }^{a}$, Damien Ségransan ${ }^{b}$, Ralf Launhardt $^{c}$, Lars B. Venema ${ }^{d}$, René Dändliker ${ }^{e}$, Jeroen A. De Jong ${ }^{a}$, Sabine Frink ${ }^{a}$, Denis Gillet $^{f}$, Saskia Hekker ${ }^{a}$, Thomas Henning ${ }^{c}$, Walter Jaffe ${ }^{a}$, Rudolf Le Poole ${ }^{a}$, Philippe Müllhaupt $^{f}$, Koji Murakawa ${ }^{d}$, Francesco Pepe ${ }^{b}$, Didier Queloz ${ }^{b}$, Laurent Sache ${ }^{g}$, Johny Setiawan $^{c}$, Danuta Sosnowska ${ }^{b}$, Rolf Wuethrich ${ }^{g}$

${ }^{a}$ Leiden Observatory, P.O. Box 9513, 2300 RA Leiden, The Netherlands

${ }^{b}$ Observatoire de Genève, 51 Ch. des Maillettes, 1290 Sauverny, Switzerland

${ }^{c}$ Max-Planck Institut für Astronomie, Königstuhl 17, 69117 Heidelberg, Germany

${ }^{d}$ ASTRON, P.O. Box 2, 7990 AA Dwingeloo, The Netherlands

${ }^{e}$ Institut de Microtechnique, Université de Neuchâtel, Rue A.-L. Breguet 2, 2000 Neuchâtel, Switzerland

${ }^{f}$ Laboratoire d'Automatique, School of Engineering, EPFL, 1015 Lausanne, Switzerland

${ }^{g}$ Laboratoire de Systèmes Robotiques, EPFL, ME3 Ch. des Machines, 1015 Lausanne, Switzerland

\begin{abstract}
A search for extrasolar planets using the ESO VLTI PRIMA facility will become feasible in 2007. An astrometric accuracy of 10 micro-arcseconds will allow us to detect sub-Uranus mass planets $\left(\mathrm{M}_{\text {Uranus }}=14.5 \times \mathrm{M}_{\text {Earth }}\right)$ around the most nearby stars, as well as to conduct a planet search around stars of different ages. Most of the PRIMA hardware subsystems are currently being developed by industry. At the same time a scientific Consortium has formed that will deliver the differential delay lines and astrometric software for PRIMA to ESO.

In this paper we describe the planned efforts by the Consortium related to the "PRIMA astrometry operations and software". These activities include an overall "PRIMA astrometry error budget", a "PRIMA astrometry calibration and observation strategy", the "PRIMA astrometry observation preparation tools" and the "PRIMA astrometry data reduction tools". We describe how all these components fit together in an overall approach to the flow of knowledge within the project. First by quantifying the fundamental limits of the VLTI infrastructure and the astronomical sources under study. Followed by elimination or suppression of the errors through either a hardware change to the system, software control of the system, or a proper calibration and observation strategy.

The ultimate goal is being able to calibrate all PRIMA astrometric data acquired over the full lifetime of PRIMA (5 to 10 years) to a uniform accuracy of 10 micro-arcseconds. This will allow identification of long-term trends in the astrometric parameters due to planetary companions around nearby stars and to determine the distances and proper motions for the selected sources.
\end{abstract}

Keywords: Interferometry, astrometry, VLTI, PRIMA, extrasolar planets, software

\section{INTRODUCTION}

\subsection{Extrasolar planets}

The first indirect detection of an extrasolar planet orbiting a main sequence star was made with the method of radial-velocity variations (Ref. 1). This prompted many other attempts to search for more planetary systems. About 120 extrasolar planets have been found with the radial-velocity method so far, with masses that span the range from the sub-Saturn mass region up to several times the mass of Jupiter, just below the brown dwarf mass range. Alternative techniques to detect the presence of extrasolar planets seem to be very promising. Among these techniques are high accuracy photometry from space to detect the occultation of a star by a planet in

Send correspondence to bakker@strw.leidenuniv.nl 
Table 1. Estimate of the maximum distances for which the astrometric signatures of planets could be detected with PRIMA.

\begin{tabular}{lrcc}
\hline \hline Planet & $\begin{array}{r}\text { full orbit } \\
\text { period } \\
\text { dyears] }\end{array}$ & $\begin{array}{c}\text { 3 year sampling of full orbit } \\
{[\mathrm{pc}]}\end{array}$ & $\begin{array}{c}\text { distance } \\
{[\mathrm{pc}]}\end{array}$ \\
\hline Earth & 1.00 & 0.3 & 0.3 \\
Jupiter & 11.87 & 480 & 255 \\
Saturn & 29.63 & 270 & 55 \\
Uranus & 84.66 & 84 & 6 \\
Warm Uranus & 6.00 & 14 & 14 \\
Hot Jupiter & 4 days & 5 & 5 \\
\hline \hline
\end{tabular}

the line of sight, gravitational microlensing, and astrometric detection of extrasolar planets through the reflex motion of its parent star. Astrometry allows the determination of the full set of orbital parameters of the system given a sufficient dense sampling of the astrometric parameters over time. With an accurate estimate of the mass of the star this gives the mass of the planet. With an astrometric accuracy of 10 micro-arcseconds, we can detect sub-Uranus mass planets around the most nearby stars. In order to detect an Earth mass planet around the most nearby stars, an astrometric accuracy below 1 micro-arcsecond would be required.

\subsection{Astrometry}

\subsubsection{VLTI/PRIMA}

Several astrometric projects are underway which have the goal to identify planets around main sequence stars. Among these are the Space Interferometry Mission (SIM, expected launch date December 2009) and the PhaseReferenced Imaging and Micro-arcsecond Astrometry (PRIMA) facility. At the European Southern Observatory (ESO) Very Large Telescope Interferometer (VLTI), at Mount Paranal in Chile, the PRIMA facility holds the challenge to astrometrically detect extrasolar planets around nearby stars. PRIMA is a dual feed system that has been designed, amongst others role, to obtain 10 micro-arcseconds relative astrometry between two sources (Ref. 2). It is currently in the process of development of the subsystems. Integration in the VLTI infrastructure will most likely start in early 2006. This will lead to first light at the end of 2006 and first science in 2007 . Since astrometry is most sensitive to planets in wide orbits, the smallest possible planets can only be identified after observing for several years at least. Therefore first scientific results with PRIMA in astrometry mode will most likely not be on extrasolar planets, but on short period binaries.

Uranus has an orbital period of 84 years with a semi-major axis of 19.28 AU. The maximum astrometric signal for a face on system, observed for the duration of half an orbital period, will be in excess of 10 micro-arcseconds for systems closer than 84 pc (see Table 1 and Fig. 1). For a reasonable observing period of 3 years with PRIMA, detection of an astrometric signal due to a Uranus mass planet requires the system to be closer than $6 \mathrm{pc}$. Along the same line Jupiter could be detectable with PRIMA for distances from 255 pc (for a 3 year sampling) up to 480 pc (for 5.9 years sampling).

Table 1 and Fig. 1 show that the detection of Earth mass planets will not be possible with PRIMA. Detection of Jupiter and Saturn mass planet is well within reach as the number of potential sources within a 55 pc volume is sufficiently large. Detection of Uranus mass planets is possible, although the number of potential systems is modest within a 6 pc volume.

\subsubsection{Astrometric parameters}

In this section we will take a look at how many parameters we have to fit for in the various observing scenarios: a science source/reference source pair, a source with a known RV companion against a reference source pair, and a visual binary system. 
full orbit

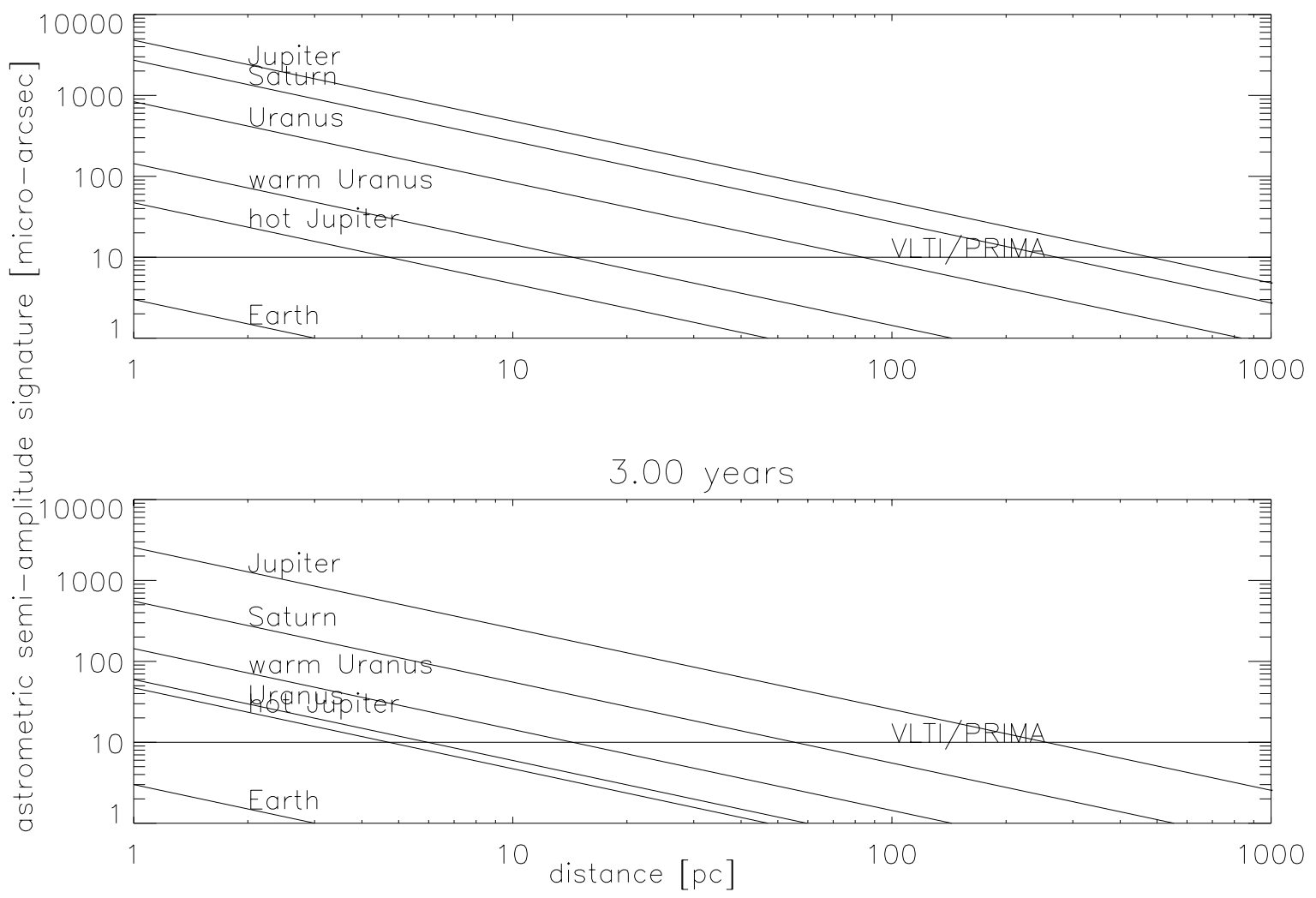

Figure 1. Detection limit of Solar system planets with VLTI/PRIMA. Top panel when the full orbit can be sampled (observing period larger than orbit). Lower panel when the orbital period is longer than the observing period of 3 years. In this case only part of the orbit is measured.

The orbit of a planet around a star can be fully characterized by seven orbital parameters (Period $P$, time of periastron passage $T_{0}$, longitude of periastron $\omega$, eccentricity $e$, mass function $f(m)$, inclination $i$, and ascending node $\Omega$ ). In the case of a known RV companion, five of these orbital parameters are known already (although maybe sometimes not with the required accuracy), so that the only two additional parameters to fit for in the astrometric orbit are inclination and ascending node.

The standard five astrometric parameters are mean positions in right ascension and declination, proper motions in right ascension and declination, and the parallax. However, since we are only doing relative astrometry, in principle we would only need to fit for one set of five astrometric parameters when we observe a science/reference source pair, against an arbitrary zero point as given e.g. by the reference source (i.e., instead of mean positions in right ascension and declination for both sources we only need to know the separation and the position angle), at least to first order. Looking at higher orders reveals that instead both parallaxes are needed, since the parallax ellipses are oriented slightly differently for sources separated by a small angle on the sky, so that we have to fit for six astrometric parameters for the case of precise relative astrometric accuracy.

Thus, in the case when observing a science and a reference source pair with no a priori knowledge of the putative companion, the astrometric fit would have 12 free parameters: seven orbital parameters and six relative astrometric parameters. However, if the companion was detected already in radial velocities, this number would be reduced by five for a total of eight parameters to fit for (two orbital ones and six relative astrometric parameters).

In the case of a visual binary, the only unknown is the orbital motion, since the astrometric parameters are the same for both stars (those of the center of mass of the system; the difference in the astrometric parameters 
are brought about only by orbital motion around the center of mass). However, orbital motion in the visual binary might introduce another seven parameters of orbital motion, for a total of 14 parameters to fit for: seven parameters of orbital motion for each the putative companion as well as the known visual binary. Again, some of the orbital parameters of the visual binary might be known already, but most of the time probably not with sufficient precision.

The relatively large number of free parameters in the astrometric solutions make it clear that many astrometric observations will have to be taken before reasonable results can be expected from these fits.

In addition to the free parameters in the fits, input parameters are needed to model the astrometric observations with the required accuracy. E.g., the radial velocity is needed for the nearest stars to correct the astrometric measurements for the effect of perspective acceleration. If it cannot be supplied with sufficient precision, it might as well have to be another free parameter in the astrometric fit.

\subsection{Consortium}

The VLT(I) has many instruments available for the community. This community can make proposals for observing time. If a Consortium makes a contribution to expanding the scientific capabilities of the VLT(I) by e.g. building an instrument, this Consortium receives guaranteed observing time in return. In the case of PRIMA a Consortium has been formed which has the objective to speed up the development of PRIMA. It will build two sets of Differential Delay Lines (DDL) much earlier than planned (Ref. 3). In addition to this, the Consortium will develop the "PRIMA astrometry observation preparation tools" and the "PRIMA astrometry data reduction tools" to ensure the scientific productiveness of PRIMA. The requirements for the software tools will be set among others by a detailed overall "PRIMA astrometry error budget analysis" and a "PRIMA astrometry calibration and observation strategy". The Consortium has two major scientific goals:

1. Accurate determination of the orbital parameters of extrasolar planets. With a focus on systems which have previously been identified by radial-velocity surveys as having an extrasolar planet orbiting the star. This will allow the determination of the mass of the planet (and not just a lower limit);

2. Planet search covering previously uncovered parameter space along the main sequence and along the time coordinate.

The contribution of the Consortium to the VLTI infrastructure and software will also enable research in areas that are not directly related to the Consortium's own scientific goals. Examples of additional important science goals of the PRIMA astrometric facility are:

1. Motions of stars: e.g. in clusters, the Galactic Center;

2. Orbital motion of stars due to e.g. binarity;

3. Distances to interesting objects based on the observed parallax.

In addition to the astrometry mode, PRIMA will allow faint source imaging by providing a stabilized (in the K-band) fringe to the other scientific instruments at the VLTI: MIDI (8 to $12 \mu \mathrm{m})$, AMBER (J, H, and K-band), GENIE (L' band), and $2^{\text {nd }}$ generation instruments to come.

Observations with PRIMA in astrometry mode, with an accuracy of 10 micro-arcseconds, is anticipated for 2007. The efforts in the PRIMA astrometry operations and software have now been well defined (July 2004). Preliminary Design Review and Final Design Review for the "PRIMA astrometry operations and software" are planned for February and October 2005 respectively, which leads to first delivery and operations in 2006. This is in phase with the major developments of the PRIMA hardware. 


\section{FLOW OF KNOWLEDGE}

Obtaining 10 micro-arcseconds astronomy with the VLTI, uniformly calibrated over a period of 5 to 10 years, is a very ambitious goal. This requires that the developments and insights in the technical and scientific issues follow a flexible road-map. This road-map, which we will refer to as the flow of knowledge (Fig. 2) in this paper, is an attempt to understand first the basic limitations and capabilities of the instrument, given the astronomical sources under study. This is achieved through an extensive error analysis phase. The outcome of an overall astrometric error budget analysis is used as an input to a calibration and observation strategy that minimizes the impact of any of the identified error sources. Either by proposing hardware changes to the infrastructure, software algorithms to compensate the errors, or by an appropriate calibration of the errors. The strategy is a matrix which presents different alternative scenarios to calibrate and observe with PRIMA. For example one could get a very high accuracy on one object by observing it for the whole night. The efficiency of the instrument will in this case be rather low. In the next phase, a calibration and observation plan will be written based on the calibration and observation strategy. It uses the strategy matrix as input and identifies the best model given many parameters involved. These parameters will cover the range from scientific to operational and cost issues.

Once the error budget and strategy/plan have been finalized, software tools can be developed. At least two major tools are required to exploit the instrument: those that allow astronomers to prepare their observations, and those that help astronomers to reduce and analyze their PRIMA astrometric data. Software to run the instrument is of course mandatory and will be developed by ESO as part of the overall VLTI software infrastructure (Ref. 4 and 5) or were delivered to ESO by other Consortia.

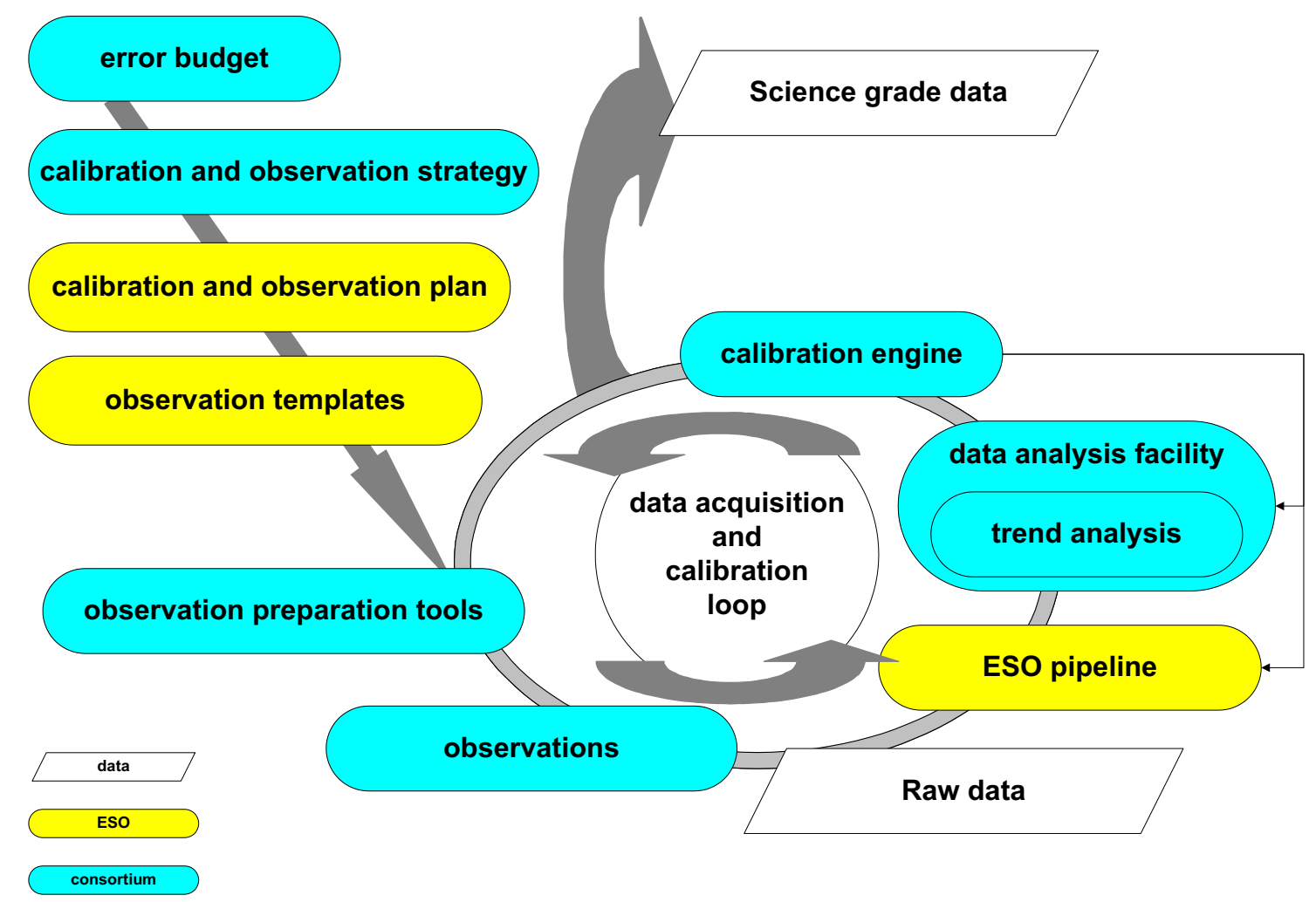

Figure 2. Flow of knowledge for the Consortium's activities related to the PRIMA astrometry operations and software.

In the next four sections we will discuss the four major components of this project:

1. PRIMA astrometry error budget; 
2. PRIMA astrometry calibration and observation strategy;

3. PRIMA astrometry observation preparation tools;

4. PRIMA astrometry data reduction tools.

\section{PRIMA ASTROMETRY ERROR BUDGET}

The error budget is the high-level tool to analyze the performance of PRIMA. It specifies the requirements for the PRIMA subcomponents and for the observation preparation tools and the data reduction tools. It also provides input to the calibration and observation strategy and plan. The error budget provides the metrics against which to verify the as-built performance of all components and procedures of the astrometric facility. We plan to construct the overall PRIMA astrometry error budget through a top-down and bottom-up process in a closed loop. First we will allocate individual errors, and estimate the required performance of the subcomponents. This will be followed by an overview of the measured error sources of the subsystems (when available). This process will be iterated through a closed loop and will be closed by a re-balancing of the error allocations and improving the calibration procedures etc., until the top-down and bottom-up error budgets match. Categories of error sources under consideration are (Ref. 3):

1. astrophysical errors;

2. atmospheric errors;

3. instrumental errors;

4. fringe sensing noise;

5. baseline errors.

\section{PRIMA ASTROMETRY CALIBRATION AND OBSERVATION STRATEGY}

The "calibration and observation strategy" is the link between the overall "astrometry error budget" and the astrometric operations at Paranal based on an "calibration and observation plan". A strategy on how to calibrate and observe with PRIMA in astrometry mode is a discussion on alternative approaches. The trade-off for each of these approaches and a matrix that compares these approaches. For explanatory purposes we will briefly describe two calibration strategies and two observation strategies.

\subsection{Calibration strategies}

Grid of visual binaries A set of 10 to 50 visual binaries could be identified. The astrometric signature from these binaries can be well predicted (although not a priori to the required accuracy) since relative proper motion errors are excluded. Hence this allows us to improve the calibration of the system and feed the calibration parameters back into the data reduction process.

Instrumental calibration The fringe sensor units will have a calibration mode that allows splitting of the light from a single source in two directions (one to represent the science source and one to represent the reference source). This technique allows calibration of systematic errors introduced by the VLTI infrastructure.

\subsection{Observation strategies}

Extremely high accuracy observations A single science source is observed for the duration of one whole night with a number of different reference sources (on one baseline). As time evolves during the night each specific reference-science source combination is repeatedly observed such that different projected baselines and position angles are probed. Also sets of reference-to-reference sources can be observed. This leads to very high astrometric measurements of a single source as error sources from the reference sources(s) can be tracked down. As a consequence the observing efficiency will be very low. Since we are interested in orbits that span several years these observations need to be repeated on a regular basis. This will decrease the efficiency of PRIMA even more. 
High observing efficiency A very high observing efficiency can be reached by observing sources when they pass the meridian (reach their highest elevation). In this case the telescopes do not have to move much from source to source and the sequence of sources to be observed (only one exposure per set of science-reference source) is determined by their right ascension. This leads to a high observing efficiency. The scientific return might not be optimal as the position angles at which the observations were obtained were not based on scientific motivations.

\section{PRIMA ASTROMETRY OBSERVATION PREPARATION TOOLS}

Several tools will need to be developed to prepare observations with PRIMA in astrometry mode. Among these are:

Ephemeris calculator A prediction of the positions of the reference and science sources based on their known orbital parameters and the errors on these parameters;

Scheduler Identification of observing conditions (epoch, baseline, position angle) that will best constrain the unknown orbital parameters, or limit the error on pre-selected orbital parameters (e.g. inclination, proper motion);

Acquisition time calculator Estimate of the amount of time required to acquire the two sources with PRIMA. This will depend on the position of the sources, their separation and magnitudes etc.; It includes setting all hardware systems, closing all control loops, and having fringe lock on the two sources.

Exposure time calculator Estimate of the required observing time to reach a specific astrometric accuracy. The integration time for each source will be based on its magnitude. For the fainter source of the two, the isoplanatic angle and the angular separation between the science and reference source will be taken into account to compute the required exposure time. The position of the fringe for the fainter sources (the optical path length difference) relative to the brighter source is the astrometric signature we are trying to measure.

\section{PRIMA ASTROMETRY DATA REDUCTION TOOLS}

The data from astrometric observations with PRIMA will have unique properties due to the measurement principle inherent in differential astrometry:

1. PRIMA will make triple differential measurements.

(a) relative angular separation on sky (up to 1 arc-minute) between the two sources;

(b) laser metrology wavelength $(\lambda=1.319 \mu \mathrm{m})$ relative to fringe tracking wavelength $(\lambda=2.2 \mu \mathrm{m})$;

(c) orbital parameters of science source relative to those of the reference source (for details see Section 1.2.2).

2. The elementary data are delay measurements with eleven significant digits. To be specific: $5 \mathrm{~nm}$ optical path length accuracy as a fraction of a 200 meter baseline.

3. The elementary delay measurements are themselves derived from multiple inputs, which are generated by different PRIMA subsystems (chiefly the two fringe sensing units and the end-to-end metrology).

4. The data have to be corrected for systematic astrophysical effects (parallax and proper motion) with amplitudes four orders of magnitude larger than the required accuracy. 
These characteristics call for a more systematic approach to data reduction than is usually applied to other instruments; PRIMA astrometric data will have to be reduced in a self-consistent manner covering an observing period comparable to the timescale expected for the astrometric signal. To realize this, the Consortium will develop the Data Analysis Facility (IDAF, Ref. 6). Benefits of a consistent comprehensive approach to data reduction are:

1. Verification of the instrumental performance. Since intermediate data products do not have a meaning at the desired level of precision, the only way to verify the instrumental performance is to check whether the residuals after the astrometric parameter fit have the expected 10 micro-arcseconds standard deviation;

2. Diagnosis and removal of systematic errors. Analysis of the dependence of the residuals on observing parameters or environmental variables (zenith angle, delay line position, outside temperature, humidity (Ref. 7), ...) may reveal systematic trends. Empirical parameterized fits of such trends can improve the data quality; systematic trends may also point towards problems in the hardware that need to be fixed;

3. Traceability of the calibration for multi-year data sets. Improved calibration procedures can easily be applied consistently to all data taken for a specific project.

To realize these ambitious goals, a three-stage data reduction process is planned:

Stage 1 On-line pipeline processing of the last exposure. This is the traditional ESO pipeline. It gives the best estimate of the astrometric parameters and does a quality check on the data received from the sub-systems.

Stage 2 On-line pipeline processing of one night of data and improved calibration (e.g. baseline).

Stage 3 Off-line processing of 5 to 10 years of data. Self calibration of the full data set. This requires the development of a dedicated Interactive Data Analysis Facility (IDAF) which delivers science grade output (Ref. 6). Requirements on the software package can be grouped in five categories. For each of these categories a brief description of the requirements will be given (for details see Ref. 6).

1. Technical performance requirements: requirements on the technical performance (e.g. amount of processing time needed);

2. Calibration requirements: tools to set the calibration model, to apply the calibration to the full data-set, to follow evolution of the calibration solution for the effective life time of PRIMA astrometry;

3. User interface requirements: this deals with the information the user provides to the system, the system provides to the user, and how the system interfaces with other systems;

4. Development standards requirements: requirements on the software standards applied for the full package, simulations and verification techniques, and product assurance (including quality control);

5. Scientific performance requirements: requirements on the overall scientific accuracy and relevance of the data reduction library.

\section{SUMMARY}

We have presented a plan on how to optimize the scientific output of the VLTI/PRIMA astrometric facility. First science in planned for 2007. This plan assumes that all the required hardware to obtain 10 micro-arcsecond astrometry will be present at that time. A (technology) road-map is presented which follows the flow of knowledge within the project to reach the final objective of 10 micro-arcsecond relative astrometry. This flow of knowledge starts with a detailed study on the astrometric error budget for PRIMA and a study on how to minimize errors through a sophisticated calibration and observation strategy and plan. These two studies are used as input to the 
specification phase of the observation preparation tools and data reduction and analysis tools. The specification phase is followed by a design and development phase aiming at first release of the software by the end of 2006 .

A key requirement for the data reduction chain will be that it allows the calibration all astrometric data of PRIMA in a self consistent manner. This requires the development of software for trend analysis in order to identify systematic errors and the generation of a calibration engine.

\section{ACKNOWLEDGMENTS}

EJB and JdJ are supported by NEVEC, a project of NOVA, the Netherlands Research School For Astronomy. SF is supported by the VICI program of the Netherlands Science Foundation (NWO). RNT is supported by the Marie Curie Program of the EU. We would like to thank ESO staff (Frédéric Derie, Françoise Delplancke, Pascal Ballester, Andreas Glindemann, Guy Monnet, Isabelle Percheron) for their kind collaboration and their interest in this project.

\section{REFERENCES}

1. M. Mayor and D. Queloz, "A Jupiter-mass companion to a Solar-type star", Nature 378, p. 355, November 1995.

2. F. Delplancke, S. Leveque, P. Kervella, A. Glindemann, and L. D'Arcio, "Phase-referenced imaging and micro-arcsecond astrometry with the VLTI", Proc. SPIE, Interferometry in Optical Astronomy; Pierre J. Lena, Andreas Quirrenbach; Eds. 4006, pp. 365-376, 2000.

3. A. Quirrenbach, T. Henning, D. Queloz, S. Albrecht, E. J. Bakker, H. Baumeister, P. Bizenberger, H. Bleuler, R. Dändliker, J. A. De Jong, M. Fleury, S. Frink, D. Gillet, W. Jaffe, S. H. Hanenburg, S. Hekker, R. Launhardt, R. Le Poole, C. Maire, P. Mullhaupt, K. Murakawa, F. Pepe, J. Pragt, L. Sache, O. Scherler, D. Ségransan, J. Setiawan, D. Sosnowska, R. N. Tubbs, L. B. Venema, K. Wagner, L. Weber, and R. Wüthrich, "The PRIMA astrometric planet search project", SPIE 2004: Astronomical Telescopes and Instrumentation 5491 this proceedings, 2004.

4. P. Ballester, A. M. Chavan, B. Cotton, V. Coude Du Foresto, A. Glindemann, C. Guirao, W. Jaffe, P. Kervella, A. Longinotti, I. Percheron, M. Peron, T. P. Duc, B. Pirenne, P. J. Quinn, A. Richichi, M. Schöller, A. Wicenec, W. R., M. Wittkowski, and S. Zampieri, "Data flow system for the very large telescope interferometer", Proc. SPIE, Astronomical Data Analysis; Jean-Luc Starck, Fionn D. Murtagh; Eds. 4477, pp. 225-233, 2001.

5. P. Quinn, M. A. Albrecht, P. Ballester, K. Banse, A. M. Chavan, P. Grosbol, M. Peron, and D. R. Silva, "VLT data flow system: from concepts to operations", Proc. SPIE, Observatory Operations to Optimize Scientific Return; Peter J. Quinn; Ed. 3349, pp. 2-9, 1998.

6. J. A. De Jong, E. J. Bakker, W. Jaffe, and S. Frink, "Preliminary software architecture for the PRIMA astrometric data analysis facility", SPIE 2004: Astronomical Telescopes and Instrumentation 5491 this proceedings, 2004.

7. S. Albrecht, E. J. Bakker, J. A. De Jong, R. N. Tubbs, J. A. Meisner, and R. Le Poole, "Calibration of temperature and relative humidity sensors for use on the VLT-Interferometer", SPIE 2004: Astronomical Telescopes and Instrumentation 5491 this proceedings, 2004. 